\title{
Ataxia, peripheral neuropathy, and anti-gliadin antibody. Guilt by association?
}

\author{
R J Lock, D S N A Pengiran Tengah, D J Unsworth, J J Ward, A J Wills
}

J Neurol Neurosurg Psychiatry 2005;76:1601-1603. doi: 10.1136/jnnp.2004.058487

Some authors contend that patients with idiopathic neurological disease who are also anti-gliadin antibody seropositive are gluten sensitive. However, anti-gliadin antibodies lack disease specificity being found in 10\% of healthy blood donors. We report a study comparing anti-gliadin antibody with other food antibodies in patients with idiopathic ataxia (20), hereditary ataxias (seven), or idiopathic peripheral neuropathy (32). Patients were HLA typed. IgA anti-tissue transglutaminase antibodies ( $\mathrm{TTG}$ ) were measured. No case was positive for $\lg \mathrm{A}$ anti-tTG making occult coeliac disease unlikely. HLA DQ2 and HLA DQ8 were found distributed equally across all patient groups and unrelated to gliadin antibody status. HLA DQ2 expressing, anti-gliadin antibody positive cases (so called "gluten ataxia") were rare in our clinics (four cases in 2 years from a population of 2 million). We conclude that coeliac disease per se is not commonly associated with either idiopathic ataxia or idiopathic peripheral neuropathy. Our study also casts doubt on the nosological status of "gluten ataxia" as a discreet disease entity. All food antibodies tested, particularly lgG, were a common finding in both ataxia and peripheral neuropathy groups. No particular food antibody was associated with any patient group. Food antibodies were equally common in hereditary ataxias. We conclude they are a non-specific finding.

n 1996, Hadjivassiliou and co-workers made the intriguing observation that patients with a variety of cryptogenic neurological disorders including ataxia, neuropathy, myelopathy, and myopathy had a strikingly high (57\%) prevalence of anti-gliadin antibodies (AGA). ${ }^{1}$ Some studies have supported these initial findings, but others reported AGA positivity in patients with idiopathic ataxia ranging from 0 to $19 \% .^{23}$ Hadjivassiliou et al subsequently found that the prevalence of AGA positivity in patients with familial ataxia was only $14 \%{ }^{4}$ and Bushara and co-workers reported AGA positivity in $27 \%$ and $37 \%$ of patients with sporadic and autosomal dominant ataxias, respectively. ${ }^{5}$ Hadjivassiliou et al subsequently focused on patients with ataxia and AGA positivity, the majority of whom carried the human leukocyte antigen (HLA) haplotype DQ2, coining the term "gluten ataxia". ${ }^{6}$ This is a potentially important observation as it suggests that simple dietary manipulation (that is, gluten restriction) provides a potential treatment for a condition hitherto thought of as inexorably progressive.

All these studies have been heavily reliant on AGA positivity as a screening tool and have inferred that the presence of these antibodies is synonymous with clinical gluten sensitivity. This is contested, as AGA are widely accepted to show poor specificity even for the prototypic gluten sensitive disorder, coeliac disease $(\mathrm{CD}) .{ }^{78}$ In contrast, in gluten sensitive enteropathy, IgA autoantibodies including anti-endomysial (AEA) and anti-tissue transglutaminase (tTG) are highly specific and sensitive. ${ }^{9} 10$

We hypothesised that the presence of AGA in neurology cases was a non-specific finding. We therefore studied the prevalence of other common irrelevant food antibodies in these patients.

\section{METHODS \\ Patients}

Over a 2 year period we prospectively investigated all newly referred patients with idiopathic or cryptogenic ataxia (27) and peripheral neuropathy (32) attending specialist clinics at Queen's Medical Centre, Nottingham and the Derbyshire Royal Infirmary. In these clinics we see 5400 new neurology referrals annually, from a background population of 2 million people. During this time we also saw 203 patients with neuropathy and 36 patients with ataxia where an underlying cause could be elucidated on routine investigation.

In the ataxic group demyelination, alcohol toxicity, prolonged use of anticonvulsant medication, and viral or paraneoplastic causes were excluded. None of the patients had evidence of multiple system atrophy type C. All the patients had an MRI of the brain and all were screened for Friedreich's ataxia and SCA 1, 2, 3, 6, and 7. During our study seven patients were discovered to have either a strong family history of ataxia (three cases) or the presence of a known genetic defect (Friedreich's ataxia, two cases; SCA 3, one case; SCA 6, one case). In the subsequent analysis, these were utilised as an internal disease control group (group 1), leaving 20 remaining cases with idiopathic ataxia (group 2).

In the acquired peripheral neuropathy group (group 3), alcohol excess, drug toxicity, paraproteinaemia, systemic vasculitis, vitamin/nutritional deficiencies, and other general medical disorders (connective tissue disorders, diabetes, etc) were excluded by appropriate investigation. In all cases the diagnosis was confirmed electrophysiologically by using standard electromyographic criteria. ${ }^{11}$ Twenty four cases were axonal and eight demyelinating in nature. These served as an external aged matched disease control group for the ataxia patients.

All patients gave informed consent and the local Ethics Committees at Derby and Nottingham approved the study.

\section{Antibody assays}

AGA (IgG and IgA) and IgA tTG were detected by enzyme linked immunosorbent assay (ELISA), according to the manufacturers instructions (Orgentec Diagnostika, Mainz, Germany). Cut off values used were those given by the

Abbreviations: AEA, anti-endomysial antibodies; AGA, anti-gliadin antibodies; CD, coeliac disease; ELISA, enzyme linked immunosorbent assay; HLA, human leukocyłe antigen; OD, optical density; PCR-SSP, polymerase chain reaction using sequence specific primers; $T$ TG, antitissue transglutaminase antibodies 
manufacturer (anti-gliadin IgG $<12 \mathrm{U} / \mathrm{ml}$, anti-gliadin IgA $<12 \mathrm{U} / \mathrm{ml}$, and $\mathrm{tTG}<10 \mathrm{U} / \mathrm{ml}$ ).

For other food antigen ELISAs, $100 \mu \mathrm{l}$ volumes of cow's milk $\beta$ lactoglobulin or chicken ovalbumin (Sigma, Poole, UK) at $20 \mu \mathrm{g} / \mathrm{ml}$ in carbonate/bicarbonate buffer were used to coat the plates overnight at $4^{\circ} \mathrm{C}$. All further incubations used $100 \mu \mathrm{l}$ volumes, at room temperature, for $\mathrm{l}$ h. All wash steps used $0.05 \%$ Tween/PBS repeated three times. After washing, patient's serum diluted to $1 / 50$ in $0.05 \%$ Tween/PBS was applied to duplicate wells. After a further wash, $100 \mu \mathrm{l}$ of either alkaline phosphatase conjugated goat anti-human IgA (Dako, Ely, UK) diluted 1 in 500 or alkaline phosphatase conjugated goat anti-human IgG (Dako) diluted 1 in 500 in 0.05\% Tween/PBS was added to each well. Sigma 104 substrate was used according to the manufacturer's instructions, and the optical density (OD) read at $405 \mathrm{~nm}$. Cut offs for in house ELISA assays, expressed as OD, were determined by the technique of probability plotting, as previously described. ${ }^{12}$ Typically, cut off values were anti-lactoglobulin IgA OD $>0.05 \mathrm{U}$, anti-lactoglobulin IgG OD>0.25 U, antiovalbumin $\operatorname{IgA} \quad \mathrm{OD}>0.05 \mathrm{U}$, and anti-ovalbumin IgG OD $>0.20 \mathrm{U}$.

Appropriate positive and negative control sera were included in all ELISA assays.

\section{Class II antigens}

DNA was extracted using the Whatman Bioscience Genomic DNA Purification System (Whatman, Brentford, UK) according to the manufacturer's instructions. HLA-DR polymerase chain reaction using sequence specific primers (PCR-SSP) was adapted from Olerup and Zetterquist. ${ }^{13}$ HLA DQ PCR-SSP was adapted from Olerup et al. ${ }^{14}$ PCR-SSP samples were subjected sequentially to five cycles of PCR $\left(96^{\circ} \mathrm{C}\right.$ for $25 \mathrm{~s}$, $70^{\circ} \mathrm{C}$ for $45 \mathrm{~s}, 72^{\circ} \mathrm{C}$ for $\left.45 \mathrm{~s}\right), 22$ cycles $\left(96^{\circ} \mathrm{C}\right.$ for $25 \mathrm{~s}, 65^{\circ} \mathrm{C}$ for $50 \mathrm{~s}, 72^{\circ} \mathrm{C}$ for $45 \mathrm{~s}$ ), and four cycles $\left(96^{\circ} \mathrm{C}\right.$ for $25 \mathrm{~s}, 55^{\circ} \mathrm{C}$ for 60 s, $72^{\circ} \mathrm{C}$ for $\left.120 \mathrm{~s}\right)$. PCR-SSP products were electrophoresed through a $1.5 \%$ agarose gel at $300 \mathrm{~V}$ for $20 \mathrm{~min}$.

\section{Statistics}

Data were analysed using the binomial test (which formally tests for a difference between the proportion of categories in a sample against a hypothesised proportion, in this case the test population and reference data), ANOVA, or Fisher's exact test, as appropriate (Analyse-it for Microsoft Excel version 1.62, Analyse-it Software, Leeds UK).

\section{RESULTS}

Table 1 shows the mean age, age range, male to female ratio, and antibody/HLA status for the three groups. Groups 2 and 3 were not significantly different in mean age $(p=0.111)$ or gender distribution $(p=0.308)$. Group 1 , found incidentally during the study, is very small and statistical analysis was considered inappropriate. The data do illustrate, however, that food antibodies of all types are also found in hereditary ataxia. A high prevalence of IgG antibodies in particular was noted, ranging from $15 \%$ to $30 \%$ in the idiopathic groups. Regarding IgA anti-tTG, no positive results were found.

\section{DISCUSSION}

We found a striking absence of serological evidence for occult CD in our cases. No case was positive for IgA anti-tTG. This is very different to the reports of other groups of $12.5-25 \%$ frequency of CD in idiopathic ataxia, ${ }^{16}{ }^{17}$ although a lower prevalence of biopsy proven CD $(2 \%)$ has been reported by one group. ${ }^{18}$ By contrast, food antibodies (wheat gliadin, hen's egg albumen, cow's milk lactoglobulin), particularly of IgG type, were common in patients with idiopathic peripheral neuropathy or ataxia. These data support and extend the findings of Abele et al showing a non-significant increase in
Table 1 Results for the three groups

\begin{tabular}{|c|c|c|c|}
\hline & $\begin{array}{l}\text { Hereditary } \\
\text { ataxia } \\
\text { (group 1) }\end{array}$ & $\begin{array}{l}\text { Idiopathic } \\
\text { ataxia } \\
\text { (group 2) }\end{array}$ & $\begin{array}{l}\text { Peripheral } \\
\text { neuropathy } \\
\text { (group 3) }\end{array}$ \\
\hline Number & 7 & 20 & 32 \\
\hline Male:female & $4: 3$ & $16: 4$ & $20: 12$ \\
\hline $\begin{array}{l}\text { Mean age (range), } \\
\text { years }\end{array}$ & $51(36-70)$ & $60(34-80)$ & $66(32-86)$ \\
\hline $\begin{array}{l}\text { Any AGA }(\lg G \\
\text { and/or } \lg A)\end{array}$ & $3(43 \%)$ & $\begin{array}{l}8(40 \%) \\
{[0.132]}\end{array}$ & 11 (34\%) \\
\hline $\begin{array}{l}\text { Any AGA and HLA } \\
\text { DQ2 positive } †\end{array}$ & $2 / 4(50 \%)$ & $4 / 8(50 \%)$ & $5 / 11(45 \%)$ \\
\hline $\begin{array}{l}\text { Any anti-lactoglobulin } \\
\text { (lgG and/or } \lg A)\end{array}$ & $2(29 \%)$ & $\begin{array}{l}6(30 \%) \\
{[0.156]}\end{array}$ & $8(25 \%)$ \\
\hline $\begin{array}{l}\text { Any anti-lactoglobulin } \\
\text { and HLA DQ2 } \\
\text { positivet }\end{array}$ & $1 / 1$ & $2 / 6(33 \%)$ & $2 / 6(33 \%)$ \\
\hline $\begin{array}{l}\text { Any anti-ovalbumin } \\
\text { (lgG and/or } \lg A \text { ) }\end{array}$ & $3(43 \%)$ & $\begin{array}{l}5(25 \%) \\
{[1.000]}\end{array}$ & $10(31 \%)$ \\
\hline $\begin{array}{l}\text { Any anti-ovalbumin } \\
\text { and HLA DQ2 } \\
\text { positive } †\end{array}$ & $2 / 3$ & $2 / 5(40 \%)$ & $3 / 9(33 \%)$ \\
\hline $\lg A$ anti-TTG & 0 & $0[1.000]$ & 0 \\
\hline HLA DQ2* $†$ & $\begin{array}{l}4 / 6(67 \%) \\
{[0.632]}\end{array}$ & $\begin{array}{l}9 / 19(47 \%) \\
{[0.894]}\end{array}$ & $\begin{array}{l}11 / 28(39 \%) \\
{[0.432]}\end{array}$ \\
\hline HLA DQ8 ${ }^{* *} \dagger$ & $\begin{array}{l}2 / 6(33 \%) \\
{[0.606]}\end{array}$ & $\begin{array}{l}4 / 19(21 \%) \\
{[0.937]}\end{array}$ & $\begin{array}{l}5 / 28(18 \%) \\
{[0.815]}\end{array}$ \\
\hline \multicolumn{4}{|c|}{$\begin{array}{l}p \text { Values (in square brackets) calculated by comparison with peripheral } \\
\text { neuropathy control population (group } 3 \text { ) except }\left({ }^{*}\right) \text { and }\left({ }^{* *}\right) \text { where } p \\
\text { values calculated against English population means of } 48.5 \text { and 18.3, } \\
\text { respectively. }{ }^{15} \\
\text { †DNA samples were not available for all cases. }\end{array}$} \\
\hline
\end{tabular}

AGA in ataxia. ${ }^{19}$ This might simply reflect an age related phenomenon or it may imply a general heightened responsiveness of the gut immune system in these individuals or some problem with gut permeability or inflammation.

Again in contrast to the findings of others, we found no over representation of HLA DQ2 or HLA DQ8 in our cases. ${ }^{6}{ }^{18}$ In other defined gluten sensitive disorders (CD, dermatitis herpetiformis), HLA DQ2 or HLA DQ8 is found in $>95 \%$ of cases. ${ }^{20}{ }^{21}$ Furthermore, in our study patients with the HLA DQ2 haplotype were no more likely to have AGA than those without (data not shown; $\mathrm{p}=0.606$ ).

Of note, we found very few patients who meet the inclusion requirements of Hadjivassiliou et al for "gluten ataxia". Whereas they describe a group of 28 patients with idiopathic ataxia in 4 years who had AGA, 23 of whom were also HLA DQ2 positive, ${ }^{6}$ we have only four patients in 2 years who meet their criteria, that is, only one third of the incidence assuming that the Neuroscience Centres in Nottingham and Sheffield serve a similar sized population. We cannot rule out ascertainment bias as a contributing factor to the differences in the two sets of data.

Our results raise a number of interesting issues with regard to the nosological status of "gluten ataxia". We disagree with the notion that AGA seropositivity per se is synonymous with gluten sensitivity either in neurological or other patient populations. Of our patients, $40 \%$ have AGA, within which must be contained the $10-15 \%$ of healthy individuals who also have AGA. Of these which have so called "gluten ataxia"? It is important to note that a similar percentage of patients with Huntington's disease also have AGA. ${ }^{22}$ Even in the classical or prototypic gluten sensitive diseases (CD and dermatitis herpetiformis), these antibodies lack specificity and alternative antibody disease markers (IgA anti-tTG, AEA) are utilised. ${ }^{7}$ An analogous situation arises with IgE class antibodies that may be detected in persons without any allergic symptoms and which in some cases disappear with time. ${ }^{23}$

Whether AGA positivity mirrors, via immune mediated mechanisms, gluten induced neurological damage is 
disputed. We contend that AGA in hereditary ataxias must be an epiphenomenon.

Our study has two major weaknesses. We did not capture all the neuropathy and ataxia cases from the background population as patients may have been referred to other hospitals in the region or not referred at all as the diagnosis was obvious (for example, diabetic neuropathy). In addition, in common with most other previous studies we have been unable to obtain antibody prevalence data on an aged matched control group (this is planned as a future study). For comparison, however, data exist for healthy younger adults, including blood donor populations, showing IgG antibodies to foods to be common. ${ }^{24} 25$ Our own (unpublished) data (blood donors, $\mathrm{n}=30$ ( 15 male), median age 40) show AGA in five (17\%), anti-ovalbumin in seven $(23 \%)$, and anti-lactoglobulin in three $(10 \%)$.

The most appealing aspect of the "gluten ataxia" hypothesis is that it offers the prospect of an exclusion diet as a realistic therapeutic possibility. ${ }^{26}$ This non-blind study needs confirmation from other centres. Our data so far do not support the view that idiopathic ataxia is a gluten related disorder. This would appear to be a case of guilt by association based on the finding of a non-specific serological marker (AGA).

\section{Authors' affiliations}

R J Lock, D J Unsworth, J J Ward, Immunology and Immunogenetics, North Bristol NHS Trust, Southmead Hospital, Bristol, UK D S N A Pengiran Tengah, A J Wills, Department of Neurology, Queen's Medical Centre, Nottingham, UK

This study was funded by the Stanhope Trust, Derbyshire Royal Infirmary, the Showering Fund, North Bristol NHS Trust, and the Immunology Research Fund, North Bristol NHS Trust. The funding sources had no role in study design, in the collection, analysis, and interpretation of data, in the writing of the report, or in the decision to submit the paper for publication.

Competing interests: none declared

Correspondence to: Dr A J Wills, Department of Neurology, Queen's Medical Centre, Nottingham, UK; adewills61@hotmail.com

Received 9 November 2004

Revised version received 1 February 2005

Accepted 7 March 2005

\section{REFERENCES}

1 Hadjivassiliou M, Gibson A, Davies-Jones GA, et al. Does cryptic gluten sensitivity play a part in neurological illness? Lancet 1996;347:369-71.
2 Combarros O, Infante J, Lopez-Hoyos M, et al. Celiac disease and idiopathic cerebellar ataxia. Neurology 2000;54:2346.

3 Abele M, Burk K, Schols L, et al. The aetiology of sporadic adult-onset ataxia. Brain 2002;125:961-8.

4 Hadjivassiliou M, Grunewald R, Sharrack B, et al. Gluten ataxia in perspective: epidemiology, genetic susceptibility and clinical characteristics. Brain 2003; 126:685-91.

5 Bushara KO, Goebel SU, Shill H, et al. Gluten sensitivity in sporadic and hereditary cerebellar ataxia. Ann Neurol 2001;49:540-3.

6 Hadjivassiliou M, Grunewald RA, Chattopadhyay AK, et al. Clinical, radiological, neurophysiological, and neuropathological characteristics of gluten ataxia. Lancet 1998;352:1582-5

7 Wills AJ, Unsworth DJ. The neurology of gluten sensitivity: separating the wheat from the chaff. Curr Opin Neurol 2002;15:519-23.

8 Cross AH, Golumbek PT. Neurologic manifestations of celiac disease. Proven, or just a gut feeling? Neurology 2003;60:1566-8

9 Unsworth DJ. Serological diagnosis of gluten-sensitivity enteropathy. J Clin Pathol 1996:49:704-11.

10 Lock RJ, Pitcher MC, Unsworth DJ. IgA anti-tissue transglutaminase as a diagnostic marker of gluten sensitive enteropathy. J Clin Pathol 1999;52:274-7.

11 Johnsen B, Fuglsang-Frederiksen A. Electrodiagnosis of polyneuropathy. Neurophysiol Clin 2000;30:339-51.

12 Lock RJ, Unsworth DJ. Immunoglobulins and immunoglobulin subclasses in the elderly. Ann Clin Biochem 2003;40:143-8.

13 Olerup O, Zetterquist H. HLA-DR typing by PCR amplification with sequencespecific primers (PCR-SSP) in 2 hours: an alternative to serological DR typing in clinical practice including donor-recipient matching in cadaveric transplantation. Tissue Antigens 1992;39:225-35.

14 Olerup O, Aldener A, Fogdell A. HLA-DQB1 and -DQA1 typing by PCR amplification with sequence-specific primers (PCR-SSP) in 2 hours. Tissue Antigens 1993;41:119-34.

15 Haworth S, Sinnot P, Davidson J, et al. Population studies. Caucasian England normal. In: Terasaki P, Gjertson D, eds. HLA 1997. Los Angeles, UCLA Tissue Typing Laboratory, 1997:208.

16 Pellecchia MT, Scala R, Filla A, et al. Idiopathic cerebellar ataxia associated with celiac disease: lack of distinctive neurological features. J Neurol Neurosurg Psychiatry 1999;66:32-5

17 Luostarinen LK, Collin PO, Peraaho MJ, et al. Coeliac disease in patients with cerebellar ataxia of unknown origin. Ann Med 2001;33:445-9.

18 Burk K, Bosch S, Muller CA, et al. Sporadic cerebellar ataxia associated with gluten sensitivity. Brain 2001;124:1013-9.

19 Abele M, Schols L, Schwartz S, et al. Prevalence of antigliadin antibodies in ataxia patients. Neurology 2003;60:1672-3.

20 Zubillaga P, Vidates MC, Zubillaga I, et al. HLA-DQA1 and HLA-DQB1 genetic markers and clinical presentation in celiac disease. J Pediatr Gastroenterol Nutr 2002;34:548-54.

21 Balas A, Vicario JL, Zambrano A, et al. Absolute linkage of celiac disease and dermatitis herpetiformis to HLA-DQ. Tissue Antigens 1997:50:52-6.

22 Bushara KO, Nance M, Gomez CM. Antigliadin antibodies in Huntington's disease. Neurology 2004;62:132-3.

23 Sanz ML, Prieto I, García BE, et al. Diagnostic reliability considerations of specific lgE determination. J Investig Allergol Clin Immunol 1996:6:152-61.

24 Husby S, Oxelius VA, Teisner B, et al. Humoral immunity to dietary antigens in healthy adults. Occurrence, isotype and lgG subclass distribution of serum antibodies to protein antigens. Int Arch Allergy Appl Immunol 1985;77:416-22

25 Kruszewski J, Raczka A, Klos $M$, et al. High serum levels of allergen specific lgG-4 (aslgG-4) for common food allergens in healthy blood donors. Arch Immunol Ther Exp (Warsz) 1994;42:259-61.

26 Hadjivassiliou M, Davies-Jones GA, Sanders DS, et al. Dietary treatment of gluten ataxia. J Neurol Neurosurg Psychiatry 2003;74:1221-4. 